\title{
Influencia de la materia orgánica y Azotobacter nigricans en un cultivo de Stevia rebaudiana B
}

\author{
Daniel Borda-Molina ${ }^{1}$, Juan Manuel Pardo-García ${ }^{1}$, José Salvador Montaña-Lara ${ }^{1}$, \\ María Mercedes Martínez-Salgado \\ ${ }^{1}$ Departamento de Microbiología, Facultad de Ciencias, Pontificia Universidad Javeriana, Bogotá D.C., Colombia \\ ${ }^{2}$ Institute of Crop Science and Resource Conservation, Faculty of agriculture, University of Bonn, Bonn, Germany \\ *jose.montana@javeriana.edu.co
}

Recibido: 12-08-2011; Aceptado: 08-11-2011

\begin{abstract}
Resumen
Stevia rebaudiana es una planta que acumula un compuesto edulcorante no calórico conocido como esteviosido. Su cultivo tradicionalmente se fertiliza con compuestos químicos que causan daño al ecosistema, obligando a buscar alternativas orgánicas para mitigar este daño. Objetivo. Evaluar el efecto de la materia orgánica y un biofertilizante con base en Azotobacter nigricans en un cultivo de Stevia rebaudiana ubicado en el departamento de Meta, Colombia. Materiales y métodos. Se establecieron cinco tratamientos: T1 y T2 Incorporando al cultivo compost de residuos domiciliarios en concentraciones de 15 y 30 ton ha ${ }^{-1}$ respectivamente; T3 y T4 con las mismas concentraciones de compost e inoculando el biofertilizante y T5 solamente con el biofertilizante. El control correspondió a las condiciones normales de cultivo con compost Bokashi. El crecimiento vegetal se evaluó por determinación de biomasa en peso seco, producción y área foliar. El análisis fisicoquímico de suelo incluyó: porcentaje carbono orgánico, humedad y pH. Resultados. La inoculación del biofertilizante produjo un incremento en la velocidad de mineralización del compost, con \% CO final de 4,85 entre los 90 y 180 días. Se presentaron diferencias estadísticamente significativas $(\mathrm{p}<0,05)$ entre la producción de biomasa de T2 $\left(1538 \mathrm{~kg}^{-1}\right)$ y el control (477 $\left.\mathrm{kg} \mathrm{ha}^{-1}\right)$. Respecto al contenido de sólidos solubles T1, T3 y T4 presentaron los más altos valores de ${ }^{\circ} \mathrm{Brix}(12,4: 12,35$ y 12,15). Conclusiones. La concentración 30 ton ha ${ }^{-1}$ presentó los mejores rendimientos de biomasa y la aplicación del biofertilizante mostró una correlación positiva con la mineralización del compost y síntesis de glucósidos.
\end{abstract}

Palabras clave: Stevia rebaudiana, biofertilizante, mineralización, carbono orgánico.

\begin{abstract}
Influence of organic matter and Azotobacter nigricans on a Stevia rebaudiana B. plantation Stevia rebaudiana is a plant that accumulates a non-caloric sweetener compound known as stevioside. This crop is traditionally fertilized with chemicals that are harmful for the ecosystem, forcing to find organic alternatives to mitigate this damage. Objective. To study the effect of organic matter and an Azotobacter nigricans-based bio-fertilizer on a Stevia rebaudiana plantation grown in acidic soil in the Department of Meta, Colombia. Materials and methods. Five treatments were established: T1 and T2 with the application of home organic waste compost at concentrations of 15 and 30 ton ha ${ }^{-1}$. T3 and T4 with the same compost concentrations and inoculating the A. nigricans bio-fertilizer. T5 contained the bio-fertilizer alone. The control consisted of the application of the Bokashi compost under the usual conditions of cultivation. Plant growth was assessed by biomass increase measured as dry weight, production, and leaf area. The physicochemical analysis of soil included: percentage of organic carbon, water content, and $\mathrm{pH}$. Results. The inoculation of the bio-fertilizer produced an increase in the rate of mineralization of compost, reaching a final 4.85\% of OC between 90 and 180 days after inoculation. There were significant $(\mathrm{p}<0.05)$ differences between biomass production with $\mathrm{T} 2\left(1,538 \mathrm{~kg} \mathrm{ha}^{-1}\right)$ and the control $\left(477 \mathrm{~kg} \mathrm{ha}^{-1}\right)$. Regarding the soluble solid content, T1, T3 and T4 showed the highest ${ }^{\circ}$ Brix values (12.4, 12.35 and 12.15, respectively). Conclusions. The concentration of 30 ton $\mathrm{ha}^{-1}$ produced the highest biomass production and the application of the biofertilizer showed a positive correlation with compost mineralization and glucoside synthesis.
\end{abstract}

Key words: Stevia rebaudiana, bio-fertilizer, mineralization, organic carbon. 


\begin{abstract}
Resumo
Influência da matéria orgânica e Azotobacter nigricans em uma cultura de Stevia rebaudiana B. Stevia rebaudiana é uma planta que acumula um composto edulcorante não-calórico conhecido como esteviosídeo. Sua cultura é tradicionalmente fertilizada com produtos químicos que danificam o ecossistema, forçando a procura de alternativas orgânicas para reduzir esse dano. Objetivo. Avaliar o efeito da matéria orgânica e um biofertilizante com base em Azotobacter nigricans numa cultura de Stevia rebaudiana localizada no departamento de Meta, na Colômbia. Materiais e métodos. Cinco tratamentos foram estabelecidos: T1 e T2 incorporando ao cultivo composto de lixo doméstico em concentrações de 15 e $30 \mathrm{t} \mathrm{ha}^{-1}$, respectivamente; T3 e T4 com as mesmas concentrações do composto e inoculando o biofertilizante e T5 apenas com o biofertilizante. O controle correspondeu a condições normais de cultivo com composto Bokashi. O crescimento das plantas foi avaliado por estimação da biomassa em peso seco, da produção e da área foliar. A análise físico-química do solo inclui o seguinte: porcentagem de carbono orgânico, umidade e pH. Resultados. A inoculação do biofertilizante produz um aumento na taxa de mineralização do composto, com CO final de 4,85\% entre 90 e 180 dias. Diferenças estatisticamente significativa $(\mathrm{p}<0,05)$ foram observadas entre a produção de biomassa de T2 $\left(1538 \mathrm{~kg} \mathrm{ha}^{-1}\right)$ e o controle $\left(477 \mathrm{~kg} \mathrm{ha}^{-1}\right)$. Com relação ao conteúdo de sólidos solúveis T1, T3 e T4 tiveram os maiores valores de ${ }^{\circ}$ Brix $\left(12,4: 12,35\right.$ e 12,15). Conclusões. A concentração de 30 ton ha ${ }^{-1}$ apresentou o melhor rendimento de biomassa e a aplicação do biofertilizante mostrou uma correlação positiva com a mineralização do composto e a síntese de glicosídeos.
\end{abstract}

Palavras-chave: Stevia rebaudiana, biofertilizante, mineralização, o carbono orgânico.

\section{Introducción}

Stevia rebaudiana $B$. es una planta herbácea perteneciente a la familia Compositae originaria de la Sierra de Amambai en la frontera entre Brasil y Paraguay (1). Su importancia radica en la presencia en sus hojas de un compuesto edulcorante no calórico 300-350 veces más dulce que la sacarosa conocido como esteviosido $(2,3)$. Presenta múltiples utilidades medicinales entre las que se encuentran: control de la hipoglicemia, prevención de caries, reducción de problemas estomacales y de piel y reducción de la presión sanguinea $(1,4)$, lo que ubica este cultivo como una especie de interés económico (5).

En Colombia, de acuerdo con datos del Ministerio de Agricultura y Desarrollo Rural (MADR, 2011), los principales departamentos productores de Stevia rebaudiana var Morita son: Antioquia, Caldas, Casanare, Valle del Cauca y Meta con un rendimiento promedio para los últimos 4 años de $4.000 \mathrm{Kg} \mathrm{ha}^{-1}$ año ${ }^{-1}$, comparable al registrado por países productores como: Paraguay con un rendimiento de 4.300 $\mathrm{Kg} \mathrm{ha}^{-1}$ año ${ }^{-1}$, Japón con 4.000 a $6.000 \mathrm{Kg} \mathrm{ha}^{-1} \mathrm{año}^{-1} \mathrm{y}$ Canadá con $3.000 \mathrm{Kg} \mathrm{ha}^{-1}$ año $^{-1}(6,7)$. La mayor parte de la producción en estos países es comercializada en los mercados de Europa y Asia, los cuales tienen una marcada tendencia a la adquisición de productos orgánicos. Este hecho obliga a los países productores a propender por sistemas orgánicos que incluyen uso de estiércoles, compost, te biológicos, extractos vegetales ó biofertilizantes con base en microorganismos fijadores de nitrógeno o fosfato-solubilizadores.

Para el cultivo de Stevia se emplean enmiendas como el compost producido a partir de los residuos vegetales del cultivo, o de mezclas de materiales, como residuos de plaza, y abonos tipo Bokashi (13), que resultan ser buen acondicionador del suelo, mejorando su estructura, porosidad, capacidad de retención de agua e intercambio catiónico (14, 15). Además disminuye la incidencia de hongos fitopatógenos como Septoria sp (15) por la producción de sustancias húmicas (ácidos húmicos y fúlvicos) que interfieren en el crecimiento micelial $(16,17)$.

Algunos microorganismos con capacidad para fijar nitrógeno atmosférico como: Azospirillum sp., y Azotobacter sp. han sido empleados como biofertilizantes en varios cultivos incluido Stevia rebaudiana (30). Las bacterias pertenecientes al género Azotobacter, subclase $\gamma$-Proteobacteria, poseen una alta capacidad fijadora de nitrógeno como consecuencia de su asociación libre con la planta. De acuerdo con la FAO, 2005 , la capacidad fijadora es del orden de 0,5 a $1 \mathrm{Kg} \mathrm{ha}^{-1}$ año (8). La conversión biológica del gas nitrógeno a amonio es catalizado por el complejo metalo-enzimático nitrogenasa, que es codificado por los genes nifH y nifDK $(8,9)$. El genero Azotobacter se encuentra ampliamente descrito en suelos tropicales asociado a la rizósfera de plantas de maíz donde incrementa la producción de fitohormonas (Ácido Indol Acético, Acido Giberélico y Ácido Abscicico) (9). También se ha reportado que la biofertilizacion de cultivos de trigo con Azotobacter sp. incrementa la síntesis de sustancias antifúngicas (11) y la producción de granos (10). A. nigricans ha sido utilizado como biofertilizante en cultivos de frijol y arroz en México, produciendo un incremento en la asimilación del nitrógeno y como consecuencia mayor rendimiento en biomasa (12). En Colombia se ha encontrado asociada a la rizósfera de cultivos de brócoli, zucchini, tomate, coliflor y espinaca en el departamento de Boyacá (8).

Las condiciones ambientales favorables de los llanos orientales de Colombia han permitido el establecimiento del cultivos de Stevia rebaudiana, Sin embargo, la baja 
fertilidad del suelo consecuencia de un pH ácido, el bajo contenido de materia orgánica, las altas concentraciones de hierro y aluminio y baja capacidad de intercambio catiónico hacen necesario establecer sistemas orgánicos adecuados para su producción. En este trabajo se evaluó la influencia de un material compostado elaborado a partir de residuos orgánicos domiciliarios en una plantación de $S$. rebaudiana, así como el efecto de la aplicación de un biofertilizante basado en Azotobacter nigricans.

\section{Materiales y métodos}

\section{Localización y muestreo de suelo}

El cultivo de Stevia rebaudiana se estableció en el municipio de Puerto López, Colombia, finca El Mirador: 0406 Latitud Norte y $72^{\circ} 50^{\prime}$ Longitud Oeste, Temperatura $27^{\circ} \mathrm{C}$, precipitación promedio: 2000 mm, HR: 75\%. Se realizó un muestreo aleatorio simple del suelo de la finca a una profundidad entre $10-20 \mathrm{~cm}$ (18) y de dos tipos de compost (residuos de plaza y Bokashi) para los análisis fisicoquímicos y microbiológicos (18).

\section{Análisis microbiológico y fisicoquímico del suelo}

El análisis microbiológico, se realizó con base en el método de recuento en placa, a partir de diluciones seriadas (18) utilizando los medios PDA (Merck ${ }^{\circledR}$, Germany) para hongos, agar avena (18) para actinomicetes, Plate Count (Merck ${ }^{\circledR}$, Germany) para bacterias heterótrofas totales y Ashby suplementado con benzoato: $\mathrm{CaCO}_{3} 5 \mathrm{~g} / \mathrm{L} ; \mathrm{NaCl} 0,2 \mathrm{~g} / \mathrm{L}$; $\mathrm{K}_{2} \mathrm{PO}_{4} 0,2 \mathrm{~g} / \mathrm{L} ; \mathrm{MgSO}_{4^{*}} 7 \mathrm{H}_{2} \mathrm{O} 0,2 \mathrm{~g} / \mathrm{L} ; \mathrm{CaSO}_{4} 0,2 \mathrm{~g} / \mathrm{L} ;$ Agar agar $15 \mathrm{~g} / \mathrm{L}$ y benzoato $5 \mathrm{~g} / \mathrm{L},(19)$, para bacterias diazótrofas. Adicionalmente, se realizó un análisis fisicoquímico completo del suelo que incluyó: $\mathrm{pH}$, humedad, y elementos en solución de suelo (N, P, K, Ca, Mg, S, Fe, Mn, Cu, Zn, B y Na). Al final del experimento (180 días) se realizó un análisis del contenido foliar de N, P, K.

\section{Producción del inoculante bacteriano e inoculación en el cultivo de $S$. rebaudiana.}

Un aislamiento identificado como Azotobacter nigricans obtenido de suelos del cultivo de Stevia (20) fue utilizado para producir el biofertilizante por fermentación discontinua (60\% de VET, $120 \mathrm{rpm} ., 32^{\circ} \mathrm{C} / 24 \mathrm{~h}$ ) en un biorreactor de 2L empleando medio a base de: leche descremada estéril al $0,5 \%(\mathrm{p} / \mathrm{v})$ y peptona de carne al $0,3 \%(\mathrm{p} / \mathrm{v})(20)$. La lectura de UFC/ml se realizó empleando la técnica de microgota de acuerdo al método propuesto por Collins (1989) en medio nutritivo (Merck ${ }^{\circledR}$, Germany).

\section{Incorporación de materia orgánica e inoculación del biofertilizante}

La incorporación de la materia orgánica se realizó en el suelo 30 días antes del trasplante de los esquejes de $S$. rebaudiana para facilitar su estabilización (6). Adicionalmente, por sugerencia del cultivador, se hizo una aplicación de cal dolomita a todos los tratamientos. Posteriormente las plantas fueron ubicadas en eras de $50 \mathrm{~m}$ por $1,20 \mathrm{~m}$ con distancia de siembra de $0,2 \mathrm{~m}$ entre plantas y $0,25 \mathrm{~m}$ entre eras (21). El biofertilizante fue aplicado por aspersión después del trasplante, en la zona de influencia de la rizósfera, utilizando $20 \mathrm{~L} / \mathrm{ha}$ de un inóculo con una concentración final de $10^{9} \mathrm{UFC} / \mathrm{ml}$, (18). La descripción de los 5 tratamientos y el control se presentan en la tabla 1.

Tabla 1. Tratamientos aplicados al suelo del cultivo de S. rebaudiana en el municipio de Puerto López, Colombia.

\begin{tabular}{cl}
\hline T/to & \multicolumn{1}{c}{ Descripción } \\
\hline Control (-) & Suelo bajo condiciones normales de cultivo \\
T1 & $\begin{array}{l}\text { Suelo con compost residuos domiciliarios } \\
\text { concentración } 15 \text { ton/ha. }\end{array}$ \\
& $\begin{array}{l}\text { Suelo con compost residuos domiciliarios } \\
\text { concentración 30 ton/ha. }\end{array}$ \\
T2 & $\begin{array}{l}\text { Suelo con compost residuos domiciliarios } \\
\text { T3 ton/ha. Inoculado con } \text { A. nigricans }\end{array}$ \\
T4 & $\begin{array}{l}\text { Suelo con compost residuos domiciliarios } \\
\text { 30 ton/ha. Inoculado con } \text { A. nigricans }\end{array}$ \\
& $\begin{array}{l}\text { Suelo con compost tipo Bokashi inoculado } \\
\text { T5 }\end{array}$ \\
\hline
\end{tabular}

Durante el cultivo se realizaron 3 podas de formación a los 30, 60 y 120 días, y la producción se llevó hasta el primer pico a los 180 días (6).

\section{Evaluación de variables fisicoquímicas y agronómicas}

Las variables fisicoquímicas y de crecimiento fueron evaluadas cada 30 días hasta los 180 días. Se determinó porcentaje de carbono orgánico (\%CO), $\mathrm{pH}$ y porcentaje de humedad (22). El área foliar empleando el software Image ${ }^{\circledR}$ de dominio público, diseñado por (National Institutes of Health, USA). El peso seco se determinó a partir de $20 \mathrm{~g}$ de material fresco (23) y el peso de producción con base en el peso seco total en cada tratamiento. Finalmente se realizó 
la cuantificación de sólidos solubles totales expresada en ${ }^{\circ}$ Brix, siguiendo el protocolo descrito por Kovylyaeva et al. (2007) (17).

\section{Análisis de resultados}

Para todas las variables evaluadas se realizó un análisis de varianza $(\mathrm{p} \leq 0,05)$ mediante el software Statistics ${ }^{\circledR}$ y se probaron los supuestos mediante ANOVA para datos de $\mathrm{pH}$, número de rebrotes, concentración de glucósidos ( $\left.{ }^{\circ} \mathrm{Brix}\right)$ y Kruskall Wallis para \% MO y \% CO, peso seco, área foliar, porcentaje de humedad. Se estableció como hipótesis: $\mathrm{H}_{\mathrm{o}}=$ las medias de todos los tratamientos son iguales $\mathrm{H}_{\mathrm{i}}=$ existe por lo menos una media de los tratamientos diferente de las otras.

\section{Resultados}

\section{Análisis microbiológico y fisicoquímico del suelo}

Se obtuvieron recuentos iniciales promedio de $10^{9} \mathrm{UFC} / \mathrm{g}$ para bacterias heterótrofas totales, $10^{7} \mathrm{UFC} / \mathrm{g}$ para actinomicetes y $10^{6} \mathrm{UFC} / g$ para hongos. Solo se presentó un descenso significativo $(\mathrm{p} \leq 0,05)$ en las poblaciones bacterianas al final del ensayo (180 días) en todos los tratamientos. En el caso de actinomicetes y hongos no se presentaron cambios significativos $(\mathrm{p} \leq 0.05),($ Tabla 2$)$.

De acuerdo con el análisis fisicoquímico del suelo en el día 0 (Tabla 3) se determinó un pH de 5,16 valor que se encuentra por debajo del rango óptimo reportado (5.5 a

Tabla 2. Recuento de microorganismos del suelo del cultivo de S. rebaudiana al inicio (día 0) y final del ensayo (día 180)

\begin{tabular}{|c|c|c|c|c|c|c|c|}
\hline \multirow{3}{*}{ Tipo de microorganismo } & \multirow{3}{*}{$\begin{array}{c}\text { Recuento } \\
\text { Día } 0\left(\mathbf{x 1 0}{ }^{7}\right) \\
\text { Suelo nativo }\end{array}$} & \multirow{2}{*}{\multicolumn{6}{|c|}{$\begin{array}{c}\text { Recuento } \\
\text { Día } 180\left(\times 10^{7}\right)\end{array}$}} \\
\hline & & & & & & & \\
\hline & & Control & T1 & $\mathbf{T} 2$ & $\mathbf{T 3}$ & $\mathbf{T 4}$ & T5 \\
\hline Bacterias & 2200 & 13 & 37 & 25 & 72 & 430 & 64 \\
\hline Hongos & 3 & 1,6 & 9 & 14 & 4 & 6 & 0,04 \\
\hline Actinomicetes & 0,27 & 0,015 & 0,6 & 0,022 & 0,07 & 0,08 & 0,015 \\
\hline
\end{tabular}

Tabla 3. Análisis fisicoquímico de suelo del cultivo de $S$. rebaudiana, en el municipio de Puerto López, Colombia.

\begin{tabular}{cccccccc}
\hline $\begin{array}{c}\text { Análisis } \\
\text { (mg Kg }\end{array}$ & $\begin{array}{c}\text { Inicio del } \\
\text { cultivo }\end{array}$ & \multicolumn{7}{c}{ Día 180 } \\
\cline { 3 - 8 } & & (control) & $(\mathrm{T} 1)$ & $(\mathrm{T} 2)$ & $(\mathrm{T} 3)$ & $(\mathrm{T} 4)$ & $(\mathrm{T} 5)$ \\
\hline $\mathrm{NO}_{3}$ & 1,8 & 3,9 & 3,6 & 5,1 & 4,1 & 4,6 & 1,3 \\
$\mathrm{NH}_{4}$ & 3,6 & 2,1 & 2,2 & 3,8 & 1,8 & 2,8 & 1,2 \\
$\mathrm{P}$ & 12 & 13 & 12 & 14 & 10 & 12 & 10 \\
$\mathrm{~K}$ & 5,8 & 12 & 15 & 18 & 15 & 36 & 12 \\
$\mathrm{Ca}$ & 13 & 40 & 34 & 46 & 29 & 56 & 41 \\
$\mathrm{Mg}$ & 2,5 & 9 & 7,9 & 7,1 & 7,9 & 11 & 7,6 \\
$\mathrm{Na}$ & 7 & 7,1 & 7,5 & 8 & 8 & 10 & 7 \\
$\mathrm{Fe}$ & 9,1 & 6,9 & 7,3 & 7,7 & 3,8 & 3,1 & 4,4 \\
$\mathrm{Cu}$ & 0,01 & 0,02 & 0,02 & 0,06 & 0,01 & 0,01 & 0,01 \\
$\mathrm{pH}$ & 5,16 & 7,99 & 7,88 & 7,18 & 7,71 & 7,96 & 7,21 \\
$\mathrm{CE} \mathrm{ds} / \mathrm{m}$ & 0,29 & 0,37 & 0,36 & 0,41 & 0,32 & 0,52 & 0,35 \\
\hline
\end{tabular}


6.5) para el desarrollo de plantas de Stevia (3). También se observó baja conductividad eléctrica $\left(0,29 \mathrm{ds} \mathrm{m}^{-1}\right)$, altos niveles de sodio; niveles sub óptimos de $\mathrm{P}$ y $\mathrm{N}$, además de una marcada deficiencia de elementos menores a excepción del hierro.

El análisis del contenido foliar de $\mathrm{N}, \mathrm{P}$ y K $\left(\mathrm{mg} \mathrm{Kg}^{-1}\right)$, mostró que en el tratamiento con 30 ton/ha de compost elaborado con residuos domiciliarios e inoculado con $A$. nigricans (T4), se obtuvo la más alta concentración de $\mathrm{P}$ y K (Tabla 4). Adicionalmente, trascurridos 60 días se observó, en todos los tratamientos, la presencia de manchas redondas pequeñas de color blanco únicamente en las hojas jóvenes, aunque en mayor proporción en el control y los tratamientos T1 y T2. Los resultados del análisis foliar mostraron que T1 y T2 presentan la menor concentración de Zn.

\section{Evaluación de variables fisicoquímicas en suelo}

En cuanto al contenido carbono orgánico $(\mathrm{CO})$, se encontraron diferencias estadísticamente significativas $(\mathrm{p} \leq 0,05)$ entre el tratamiento que contiene 30 ton $\mathrm{ha}^{-1}$ de compost $\mathrm{y}$ Azotobacter nigricans (T4) y los demás tratamientos. En general se observó un marcado descenso de los valores de
Tabla 4. Análisis foliar en el cultivo de $S$. rebaudiana, en los diferentes tratamientos en el día 180.

\begin{tabular}{ccccc}
\hline T/to & $\mathbf{N}(\%)$ & $\mathbf{P}(\%)$ & $\mathbf{K}(\%)$ & $\mathbf{Z n}\left(\mathbf{m g ~ K \mathbf { ~ g } ^ { - 1 } )}\right.$ \\
\hline Control (-) & 1,96 & 0,44 & 1,53 & 37 \\
T1 & 1,96 & 0,34 & 1,98 & 37 \\
T2 & 1,98 & 0,35 & 1,97 & 35 \\
T3 & 1,98 & 0,35 & 2,19 & 43 \\
T4 & 2,15 & 0,48 & 2,20 & 73 \\
T5 & 1,92 & 0,41 & 1,97 & 43 \\
\hline
\end{tabular}

CO entre los días 90 y 120 llegando a estar por debajo de los niveles óptimos reportados para el cultivo de $S$. rebaudiana (5 a 8\%). Posteriormente para el día 180, el tratamiento T4 presentó una disminución marcada en el contenido de CO en comparación con T5 y el control, con valores de 4,85\% y $3,02 \%$ respectivamente (Figura 1).

Con relación al pH (Figura 2a), se observó hasta el día 60 un incremento en todos los tratamientos excepto en $\mathrm{T} 4$ ( $\mathrm{p} \leq 0,05)$, debido a la aplicación de cal dolomita antes

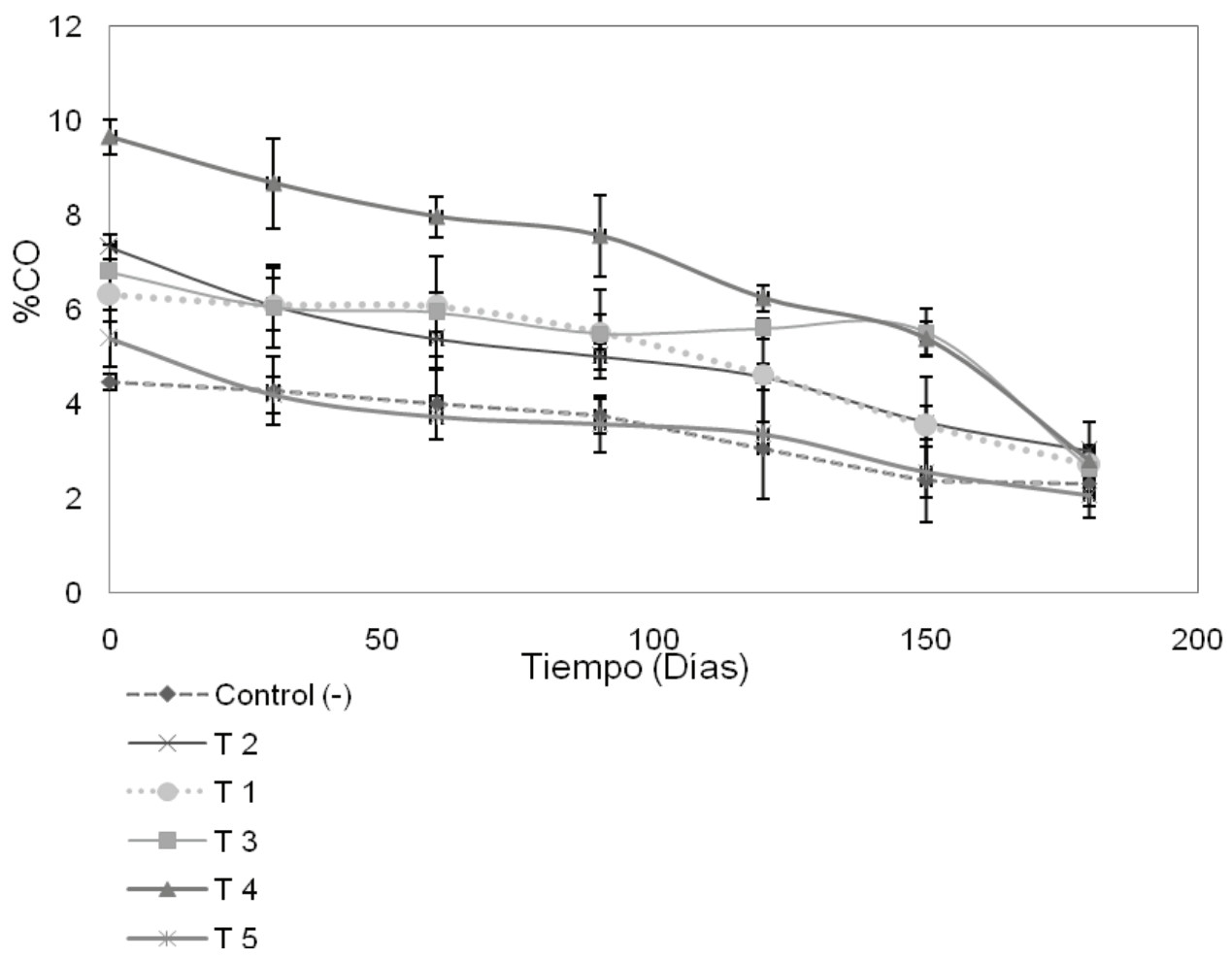

Figura 1. Variación del Carbono orgánico en los diferentes tratamientos del cultivo de S. rebaudiana en el municipio de Puerto López, Colombia. 
de la siembra e incorporación del compost al momento del trasplante. En el día 90 se registró un descenso en el valor del $\mathrm{pH}$ y posteriormente entre los días 150 y 180 se presentó un incremento, con tendencia hacia la alcalinización.

Uno de los efectos más notorios de la aplicación de la materia orgánica se evidenció por el aumento del porcentaje de humedad del suelo en todos los tratamientos entre los días 90 y 180, excepto en el control ( $\mathrm{p} \leq 0,05)$ (Figura 2b).

\section{Evaluación de variables agronómicas en el cultivo}

En general el área foliar (Figura 3a) se incrementó entre los 30 y 60 días como consecuencia de la poda de formación y posteriormente disminuyó hasta los 180. Se encontraron diferencias significativas entre tratamientos $(\mathrm{p} \leq 0,05)$, siendo el control el que presentó los resultados más dispersos. Con relación al peso seco (Figura 3b), se encontró una marcada disminución, reflejada en una baja producción entre los días 60 y 90 excepto para T1 y T2

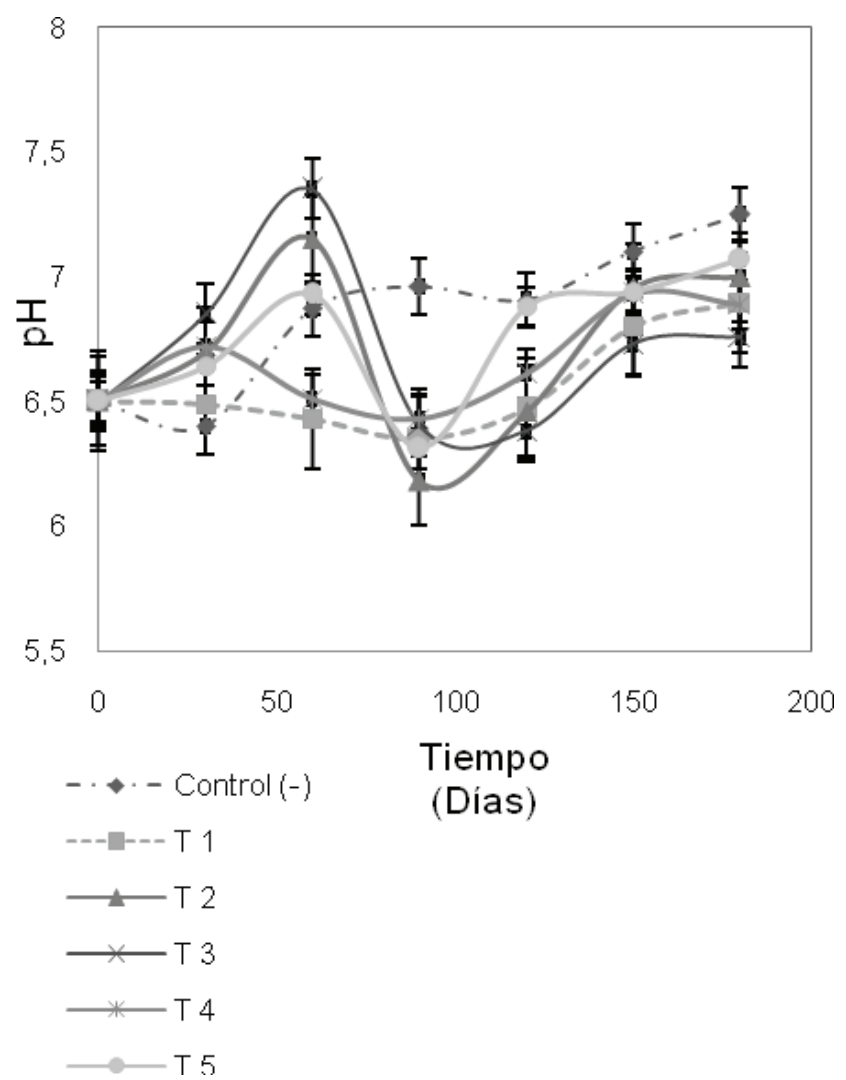

a. con diferencias estadísticamente significativas $(\mathrm{p} \leq 0,05)$. En el día 150 se obtuvieron diferencias estadísticamente significativas $(\mathrm{p} \leq 0,05)$ entre $\mathrm{T} 1 \mathrm{y}$ el control, y entre T2 y el control; siendo este último tratamiento el que se encuentra por debajo de la media general.

El peso fresco de producción, se incluyó como un estimado del total de peso tomado en cada tratamiento a los 180 días. La mayor producción se observó en T1 (1200 kg ha-1) y T2 (1538 $\mathrm{kg} \mathrm{ha}^{-1}$ ) respectivamente (Figura 4).

En cuanto a la concentración de solidos solubles en el material vegetal, se obtuvieron diferencias estadísticamente representativas $(\mathrm{p} \leq 0,05)$. El tratamiento $\mathrm{T} 1$ presentó el valor más alto $\left(12,45^{\circ}\right.$ Brix) (Figura 5) siendo superior a la media general, mientras que los tratamientos $\mathrm{T} 2 \mathrm{y}$ control presentaron valores de ${ }^{\circ}$ Brix inferiores $(11,5$ y 11,3$)$.

En cuanto a los kg de elementos/ha en peso seco, se encontró que el tratamiento que mayor cantidad de elementos extrajo del suelo fue T2 (Tabla 5), esto coincide con el hecho que fue el tratamiento que presentó la mayor producción (1538 $\left.\mathrm{kg} \mathrm{ha}^{-1}\right)$.

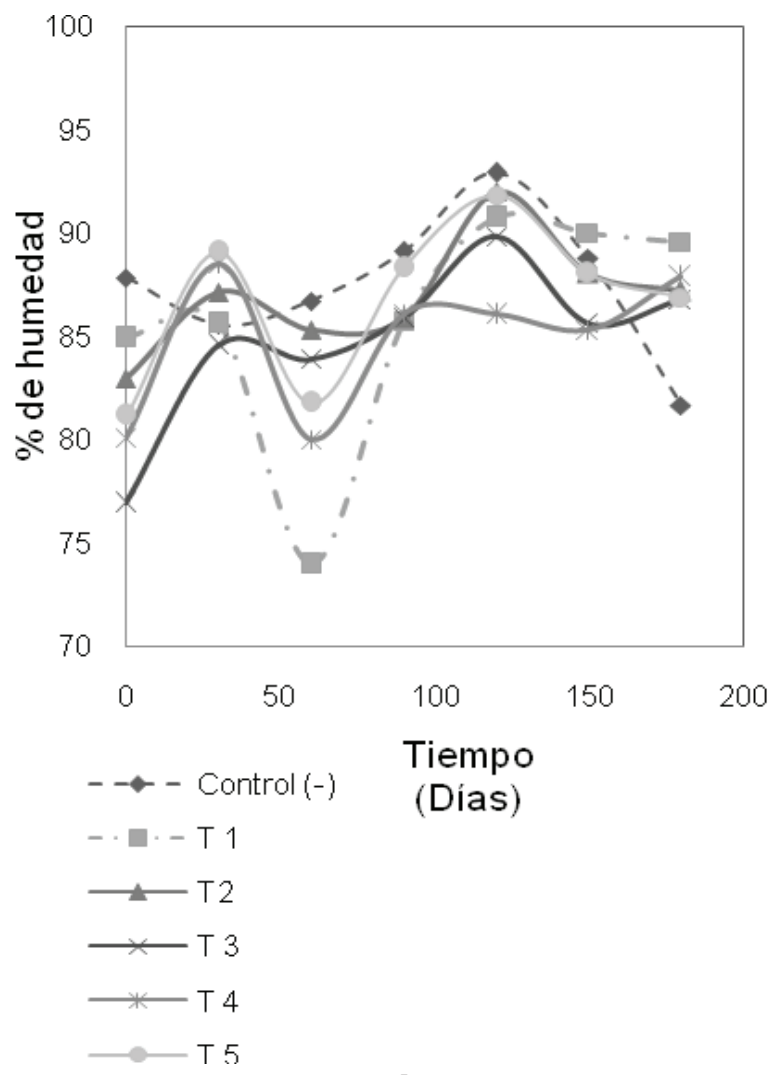

b.

Figura 2. Evaluación de variables fisicoquímicas del suelo en los diferentes tratamientos del cultivo de $S$. rebaudiana, en el municipio de Puerto López, Colombia. a. pH. b. Porcentaje de humedad. 


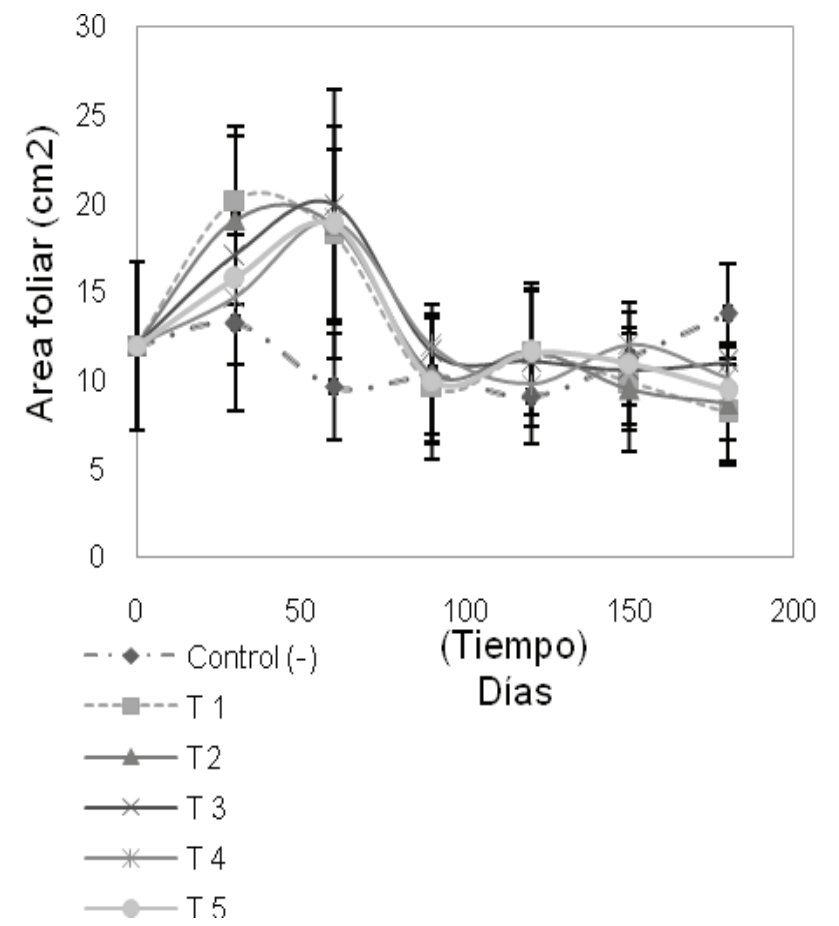

a.

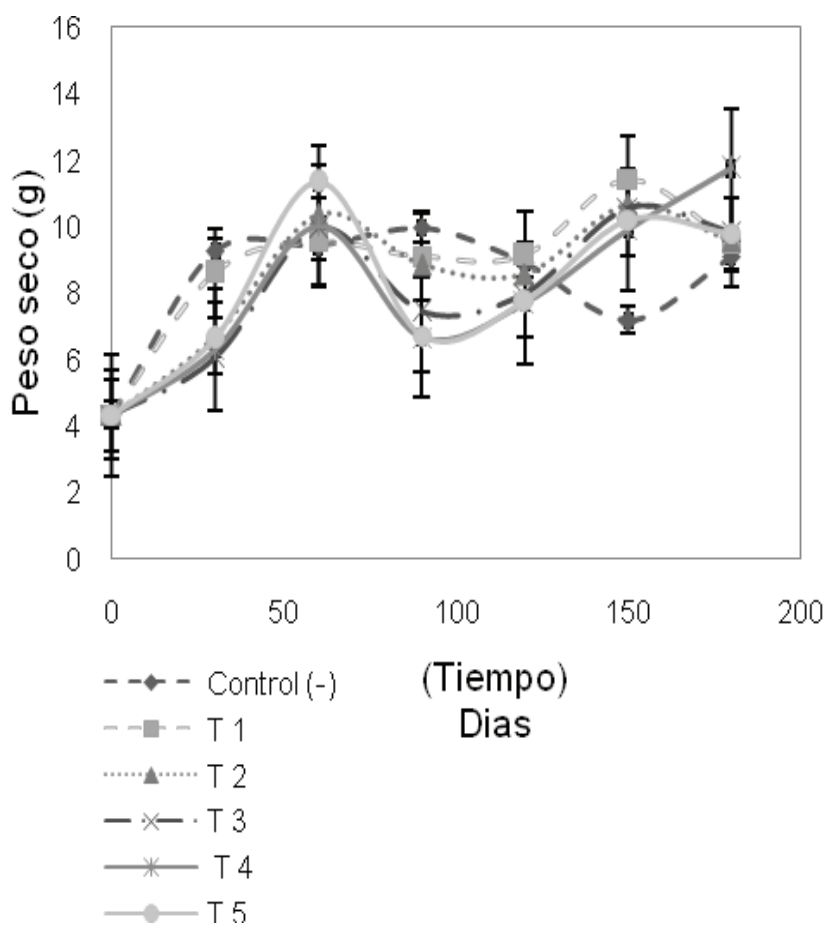

b.

Figura 3. Variables agronómicas evaluadas en el cultivo de S. rebaudiana en el municipio de Puerto López, Colombia a los 180 días a. Área Foliar. b. Peso seco.

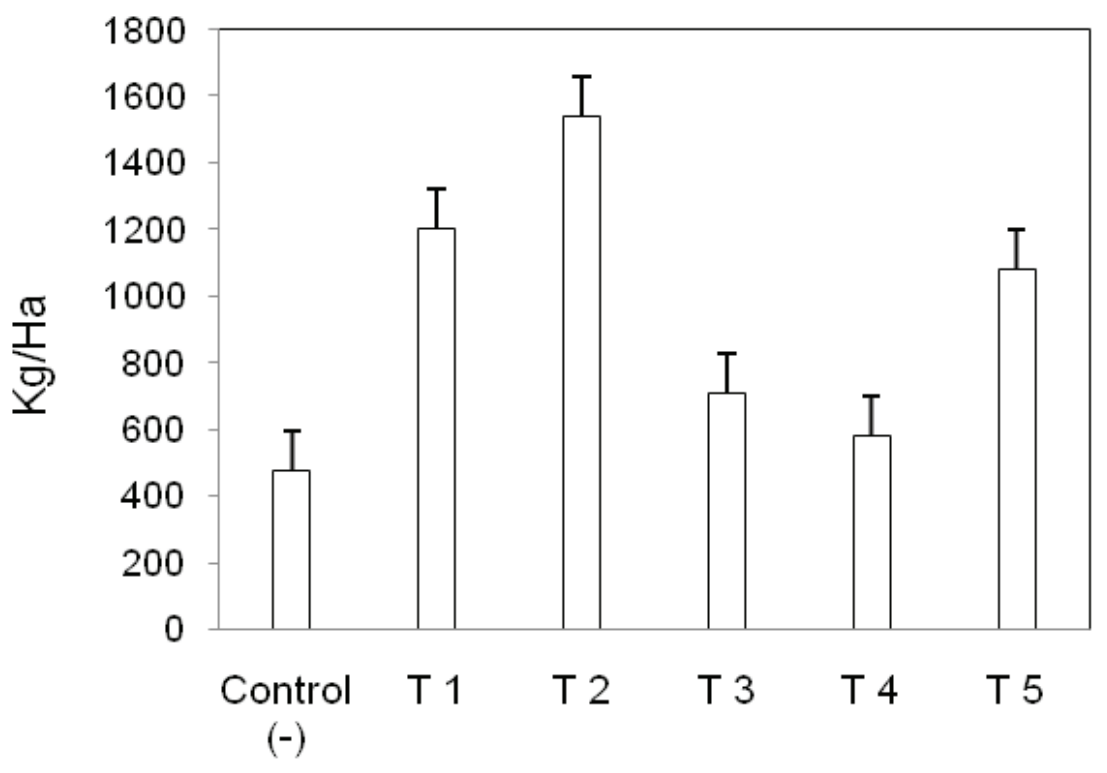

\section{Tratamientos}

Figura 4. Producción del cultivo $S$. rebaudiana en $\mathrm{Kg} / \mathrm{Ha}$ en el municipio de Puerto López, Colombia al final del estudio (180 días). 


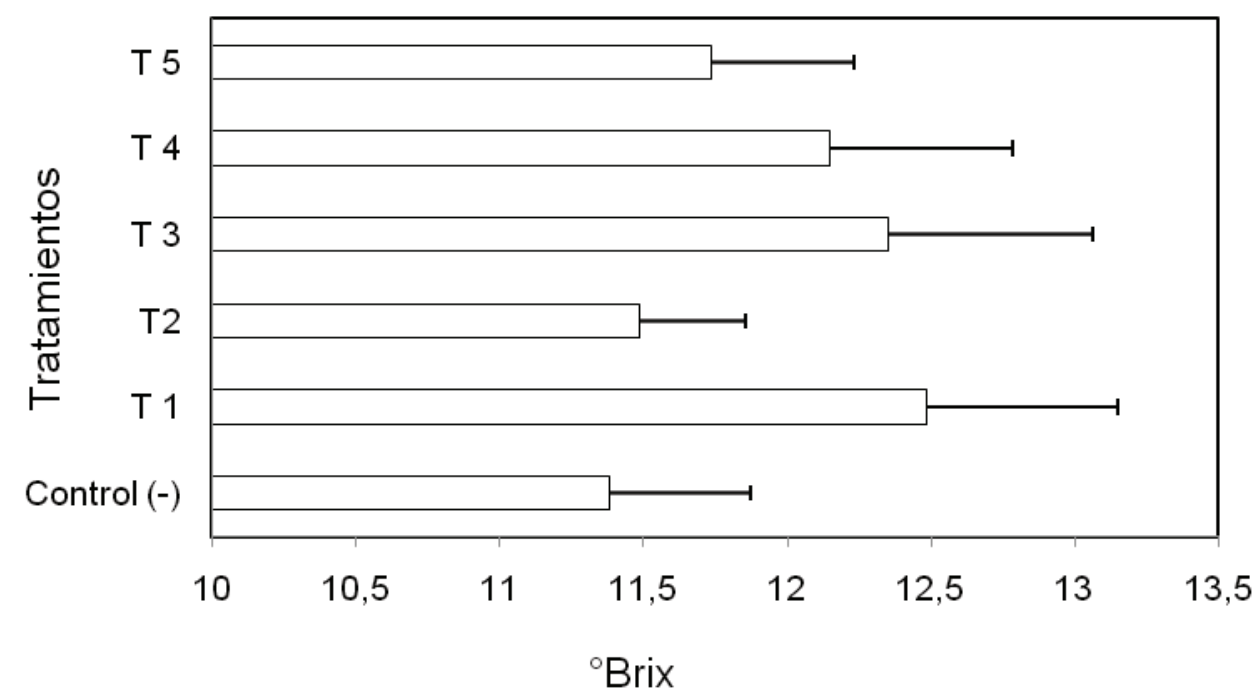

Figura 5. Sólidos solubles totales ( ${ }^{\circ}$ Brix) para el dia 180 en los diferentes tratamientos, del cultivo S. rebaudiana establecido en el municipio de Puerto López, Colombia.

Tabla 5. Kg ha ${ }^{-1}$ de N, P y K extraídos del suelo del cultivo de $S$. rebaudiana, en el municipio de Puerto López, Colombia.

\begin{tabular}{|c|c|c|c|}
\hline T/to & $\mathbf{N}\left(\mathbf{k g ~ h a} \mathbf{a}^{-1}\right)$ & $\mathbf{P}\left(\mathbf{k g ~ h a} \mathbf{a}^{-1}\right)$ & $\mathbf{K}\left(\mathbf{k g ~ h a} \mathbf{h}^{-1}\right)$ \\
\hline Control (-) & 3,890 & 0,873 & 3,036 \\
\hline $\mathrm{T} 1$ & 10,361 & 1,797 & 10,414 \\
\hline $\mathrm{T} 2$ & 12,847 & 2,271 & 12,782 \\
\hline $\mathrm{T} 3$ & 5,610 & 0,992 & 6,205 \\
\hline $\mathrm{T} 4$ & 5,034 & 1,124 & 5,151 \\
\hline $\mathrm{T} 5$ & 8,394 & 1,792 & 8,612 \\
\hline
\end{tabular}

\section{Discusión}

La aproximación empleada para determinar la influencia de la aplicación de un material orgánico y un biofertilizante en un cultivo $S$. rebaudiana consistió en la evaluación de parámetros microbiológicos, fisicoquímicos y agronómicos. El recuento de microorganismos del suelo en cada uno de los tratamientos (Tabla 2), evidenció un comportamiento dinámico y transitorio de las poblaciones microbianas debido a la incorporación del compost al cultivo. Se encuentra documentado que la enmiendas orgánicas pueden modificar condiciones fisicoquímicas del suelo como: el $\mathrm{pH}$, contenido de humedad y conductividad eléctrica, lo que a su vez determina la estructura de las comunidades microbianas (18).
La evaluación de los parámetros fisicoquímicos (Tabla 3) permitió evidenciar un bajo contenido de materia orgánica al inicio del estudio. Según Rao et al., (2005) (25) El bajo contenido de materia orgánica consecuencia del escaso aporte de biomasa encontrado en los suelos de los llanos orientales que se encuentra entre 2.2 y 3.8 ton ha $^{-1}$ año $^{-1}$ explica la media o baja fertilidad del suelo. Pregitzer (2005) (26), afirma que la disponibilidad y movilidad de los nutrientes en el suelo, están directamente relacionadas con variables ambientales como precipitación, humedad relativa y la temperatura, de manera que suelos con textura franco arenosa en regiones de alta pluviosidad tienden a perder con mayor facilidad elementos solubles y a ser más ácidos. De otra parte, bajos porcentajes de humedad pueden dificultar el flujo de masas y la difusión de nutrientes, limitando el crecimiento de la planta. Teniendo presente esto, el aporte de materia orgánica era una situación indispensable más aun considerando que al inicio del cultivo se presentaron altas precipitaciones.

Con relación al pH se observó una dinámica particular. En el tratamiento control el pH del suelo tuvo una tendencia a aumentar, hasta valores de 7.2 en el día 180. Estos valores sumados a la baja humedad podrían limitar la disponibilidad de nutrientes como P, Fe, Mn, B y Cu (18). Das et al., (2008) (30), afirma que para cultivos de Stevia una deficiencia del $\mathrm{N}, \mathrm{P}, \mathrm{K}$ y Mg, y algunos micronutrientes, conllevan a una reducción del crecimiento vegetativo en términos del área foliar y longitud de las raíces (6). En el caso del tratamiento con 15 ton ha ${ }^{-1}$ de compost (T1) no se observaron variaciones 
significativas en los valores de $\mathrm{pH}$ durante los primeros 90 a 120 días. Sin embargo a partir de este momento el pH se incrementó ligeramente hasta alcanzar un valor promedio de 6.8 en el día 180. Para los tratamientos T2, T3, T4 y T5 las variaciones fueron evidentes posiblemente como consecuencia de la incorporación de compost en mayor cantidad ó la aplicación del biofertilizante. El marcado descenso en el $\mathrm{pH}$ para estos tratamientos en el día 90 puede explicarse por la presencia de ácidos orgánicos solubles generados en el proceso de mineralización y el ácido carbónico formado por la liberación de $\mathrm{CO}_{2}$. El incremento del $\mathrm{pH}$ entre los días 150 y 180, sugiere hacer una nueva aplicación de materia orgánica después de los 90 días, con el fin de evitar problemas de deficiencias nutricionales por la no disponibilidad de nutrientes para la planta, (18).

De otra parte, el tratamiento T4 (30 ton ha ${ }^{-1}$ con A. nigricans) presentó una mayor conductividad eléctrica (dS/m 0,52) (Tabla 3) lo que puede estar influyendo en una alta mineralización, con consecuencias sobre la concentración de $\mathrm{K}$ (36 mg $\mathrm{Kg}^{-1}$ ) y P (12 mg Kg-1), macronutrientes indispensables para el crecimiento de la planta, el engrosamiento de los tallos, y la formación de rebrotes (30).

Uno de los parámetros que permite evidenciar la dinámica de la transformación de la materia orgánica es el porcentaje de Carbono orgánico. En este trabajo T4 presentó una disminución marcada de este parámetro con relación a T5 y el control (Figura 1), Este comportamiento se puede explicar por una mayor disponibilidad de nutrientes que incrementan la actividad metabólica tanto de los microorganismos presentes en el compost como el biofertilizante haciendo más eficiente el proceso de mineralización (Tabla 3 ). Se ha reportado que Azotobacter sp. al ser inoculado en compost proveniente de residuos domiciliarios, incrementa la cantidad y calidad de nutrientes, transformando un compost normal en un compost bioactivo (28), Este puede ser el caso de T4 donde la incorporación de 30 ton $\mathrm{ha}^{-1}$ y el biofertilizante, $A$. nigricans resulta en un efecto sinergístico que permite una mineralización eficiente, aumento de la biomasa de raíces y mayor disponibilidad de nutrientes para la planta lo que se traduce en aumento de la tasa de crecimiento y mayor actividad metabólica $(9,27)$.

El marcado descenso de Carbono Orgánico entre los días 120 y 180 para los tratamientos T2 y T4, sugiere una alta mineralización de la materia orgánica que depende de la cantidad de compost incorporado al cultivo inicialmente; en el caso de T4 este proceso es aún más rápido. Considerando que Stevia rebaudiana es una especie semi-perenne con un ciclo productivo de 4 años y que el proceso de descomposición y mineralización de la materia orgánica en los suelos de los llanos orientales según Rao et al., (2005) (25) ocurre de manera rápida, posiblemente es necesaria una segunda incorporación de materia orgánica a partir de los 90 días de establecido el cultivo.

La persistencia del compost en el suelo, el riego y régimen de lluvias influyen en los valores de porcentaje de humedad como consecuencia del aumento en la retención de agua en el suelo por la presencia de materia orgánica. Es importante considerar que el alto porcentaje de humedad en el suelo (Figura 2b), puede contribuir a mejorar la tasa de descomposición de la materia orgánica, así como la disponibilidad de nutrientes para la planta (29).

Los mayores contenidos de N, P, K y Zn en las hojas de Stevia en T4, soporta la hipótesis que una concentración de 30 ton ha $^{-1}$ permite a la planta una mejor asimilación de nutrientes y por lo tanto un mayor crecimiento. Tulasi (2008) recomienda aplicaciones de 50 ton ha-1 en cultivos de Stevia del norte de India mientras que Maya (2000) recomienda la incorporación de 20 ton ha ${ }^{-1}$ en cultivos colombianos, En este trabajo además de la incorporación de materia orgánica, se sugiere la inoculación de la bacteria diazótrofa A. nigricans por su capacidad para fijar de nitrógeno atmosférico, sintetizar promotores de crecimiento vegetal y solubilizar fosfato $(27,29)$.

Por otro lado la presencia de manchas redondas pequeñas de color blanco únicamente en las hojas jóvenes, sugirió inicialmente una posible deficiencia de elementos menores poco móviles como $\mathrm{Zn}, \mathrm{B}$ y $\mathrm{Cu}$ (27). El análisis foliar (Tabla 4) mostró una relación inversa entre la concentración de Zn y la presencia de manchas cloróticas, que si bien son síntoma de deficiencia en variedades de maíz, podrían también sugerirla en este cultivo. La anterior hipótesis es soportada por el hecho de que en el análisis fitopatológico no se evidenció la presencia de patógenos (hongos y bacterias) en el material vegetal, que pudieran ser la causa de la clorosis observada. Adicionalmente se encontró, que en el tratamiento con 30 ton ha $^{-1}$ de compost con A. nigricans, los contenidos foliares de $\mathrm{Zn}$ a los 180 días fueron los más altos y la cantidad de manchas cloróticas disminuyó considerablemente.

El área foliar no presentó cambios significativos entre los tratamientos, lo que permite inferir que esta variable se encuentra más relacionada con el estado fenológico de la planta $(1,5)$ y con las variaciones de la intensidad de la luz que influyen directamente en el proceso fotosintético. Estudios realizados por Rengifo et al., (2005) (1) demuestran que dos variedades diferentes de $S$. rebaudiana (Morita 1 y Morita 2) pueden ser potencializadas o nó, dependiendo de la radiación solar incidente. Sin embargo en este trabajo este aspecto no fue considerado. 
En cuanto a la extracción de nitrógeno y potasio, se pudo establecer que fue superior a la de $\mathrm{P}$ en todos los tratamientos. Los valores de N, P y K extraído para T2 fueron 12.8, 2.27 y $12.7 \mathrm{~kg} \mathrm{ha}^{-1}$ respectivamente, razón por la cual se puede afirmar que una planta de $S$. rebaudiana de 180 días, bajo las condiciones de la finca evaluada, para tener una producción que supere los $1500 \mathrm{~kg} \mathrm{ha}^{-1}$ (producción máxima registrada en este trabajo) necesita extraer las cantidades anteriormente mencionadas.

El peso seco de producción que se encontró para T1 (1200 $\left.\mathrm{kg} \mathrm{ha}^{-1}\right)$ y T2 $\left(1538 \mathrm{~kg} \mathrm{ha}^{-1}\right)$ es consistente con los valores reportados para cultivos de Stevia en Bolivia $(1144,8 \mathrm{~kg}$ ha $^{-1}$ ), Japón (1500 a $\left.1750 \mathrm{~kg} \mathrm{ha}^{-1}\right)$ y Tasmania (1100 $\left.\mathrm{kg} \mathrm{ha}^{-1}\right)(5,22)$. En el caso de T4 donde se obtuvo una producción curiosamente más baja $577,7 \mathrm{~kg} \mathrm{ha}^{-1}$ (Figura 4), la acumulación de potasio en tallos y hojas de $2,2 \%$ y de fosforo 0,48\%. Das et al. (2008), reportan cantidades de potasio acumulado en tallos y hojas de $1,7 \%$ y de fósforo $0,22 \%$ respectivamente en cultivos con una producción promedio $7500 \mathrm{~kg} \mathrm{ha}^{-1}$ en cultivos en India, esto indica que los valores dependen del tipo de suelo y clima específico en el que se encuentre el cultivo. La mayor concentración de potasio y fósforo solubles obtenida en T4 sugería una mayor producción del cultivo, sin embargo la producción alcanzada fue de tan solo $577,7 \mathrm{~kg} \mathrm{ha}^{-1}$, siendo una de las más bajas registradas. Considerando que las características fisicoquímicas observadas para el suelo en T4 son las más adecuadas para obtener valores de producción superiores, la baja producción obtenida, posiblemente se debe a que la estabilización del suelo requiere más tiempo. Seguramente en las etapas de producción posteriores, el peso seco de producción bajo estas condiciones podría incrementar como consecuencia de una mejor condición del suelo.

Con relación a la concentración de sólidos solubles totales, los tratamientos T1, T3 y T4 presentaron valores superiores al control confirmando que la incorporación de compost al suelo del cultivo de Stevia puede incrementar los niveles en las hojas, consecuencia de una mejor disponibilidad de nutrientes en el suelo y por la producción de Giberelinas por A. nigricans, que encuentran una ruta de síntesis similar al esteviosido (ruta del ácido mevalónico), excepto por la síntesis de kaureno. La biosíntesis de estos dos compuestos está separada temporal y espacialmente y depende de los mecanismos de regulación de la expresión genética que su vez se encuentran influenciados por múltiples factores, entre ellos las condiciones ambientales. Debido a que la síntesis de esteviosido se da en su mayoría en hojas viejas y la de ácido giberélico en hojas jóvenes, se ha reportado que este glucósido es sintetizado por la planta como un mecanismo para evitar una sobreproducción de ácido giberélico $(20,30)$, En este trabajo la aplicación de A.nigricans posiblemente aumente la concentración de giberelinas, activando los genes para la síntesis de esteviósidos que contribuyen al aumento de los valores de sólidos solubles totales.

Los tratamientos T1, T3, T4 que tienen la mayor concentración de glucósidos, presentan las mayores cantidades de $\mathrm{K}$ acumulado en hojas (T1: 1,98\%, T3: 2,19\%, T4: 2,2\%) (Tabla 4); lo que sugiere que la concentración de este nutriente influye en la cantidad de glucósidos almacenados. Tulasi (2008) afirma que la mejor producción de glucósidos de $S$. rebaudiana se obtiene cuando el potasio está en altas concentraciones, entendiendo que este elemento es activador de diferentes enzimas involucradas en el metabolismo, respiración y fotosíntesis.

Observando en conjunto las condiciones de suelo y la concentración de glucósidos, autores afirman que en condiciones con cantidades excesivas de sales en el medio, pueden interferir en la síntesis de metabolitos secundarios (30). En el presente trabajo, el exceso de Ca disponible $\left(13 \mathrm{~m} \mathrm{~kg}^{-1}\right)$ pudo haber afectado la síntesis de glucósidos especialmente en el tratamiento control el cual presentó el porcentaje de saturación de Ca más alto $(36,7 \%)$, este elemento tiene una función metabólica que es crítica en la eliminación de los carbohidratos y se evidencia al encontrar que el exceso de este elemento interfirió con la acumulación de glucósidos en las hojas. Este hecho está presente en el tratamiento T5, que al ser inoculado con A. nigricans, presentó una alta acumulación de glucósidos como consecuencia de un incremento en la velocidad de mineralización de la materia orgánica generando $\mathrm{CO}_{2}$, que al reaccionar con agua y en un $\mathrm{pH}$ ligeramente alcalino, precipita el calcio en forma de $\mathrm{CaCO}_{3}$ no estando disponible para la planta (24).

La aplicación del biofertilizante producido a partir de $A$. nigricans generó un efecto positivo sobre la acumulación de nutrientes (N, P y K) en las hojas de S. rebaudiana especialmente para T5, donde la cantidad de elementos ha- ${ }^{-1}$ en peso seco $8.3 \mathrm{~N}, 1.7 \mathrm{P}, 8.6 \mathrm{~K} \mathrm{~kg} \mathrm{ha}^{-1}$ fue mayor que el control y $3.8 \mathrm{~N}, 0.8 \mathrm{P}, 3.0 \mathrm{~K} \mathrm{~kg} \mathrm{ha}^{-1}$. Esta situación se debe a las propiedades que tiene el microorganismo para promover el crecimiento vegetal a través de la fijación de nitrógeno y la solubilización de P y K (10), sin tener el efecto esperado en la producción de biomasa, probablemente porque las consecuencias de su establecimiento se evidencian en una etapa posterior del cultivo.

\section{Conclusiones}

La cepa de Azotobacter nigricans inoculada en los diferentes tratamientos, incrementó la velocidad de mineralización 
del compost que se completó entre los 90 y 120 días, especialmente para $\mathrm{T} 4$ que contiene 30 ton ha $^{-1}$ de compost elaborado a partir de residuos de plaza. Bajo las condiciones de cultivo evaluadas, se recomienda hacer aplicaciones de 30 ton ha ${ }^{-1}$ de compost de residuos de plaza inoculado con A. nigricans como una alternativa para enfrentar los problemas de baja fertilidad y mantener una producción orgánica en el cultivo de Stevia.

\section{Agradecimientos}

Los autores agradecen especialmente al Centro Coordinador de la Investigación de la Federación Internacional de Universidades Católicas CCI-FIUC y a las empresas Bioagrícola del Llano y Agroestevia S.A

\section{Financiación}

Esta investigación fue realizada con el apoyo de la Federación Internacional de Universidades Católicas FIUC y el Centro Coordinador de la Investigación CCI-FIUC, en el marco del proyecto "Formación de técnicos para mejorar la calidad de suelos en países en vía de desarrollo: empleo de compost mejorado biotecnológicamente en cultivos orgánicos." ID: 000158 .

\section{Conflictos de intereses}

Los autores expresan que no existen más que intereses científicos alrededor de los resultados de esta investigación.

\section{Referencias}

1. Rengifo T, Jarma A, Aramendiz T. Aspectos fisiológicos de estevia (Stevia rebaudiana Bertoni) en el Caribe Colombiano: I. Efecto de la radiación incidente sobre el área foliar y la distribución de biomasa. Agronomía Colombiana 2005; 23 (2): 207-216.

2. Huang X, Fu J, Di D. Preparative isolation and purification of steviol glycosides from Stevia rebaudiana Bertoni using high speed counter-current chromatography. Separation and Purification Technology 2009; 71: 220-224.

3. Tamayo A, Hincapie M. El cultivo de la Estevia (Stevia rebaudiana Bert.). Corpoica-Centro de investigación La Selva. Rionegro-Antioquia-Colombia. 2005: 16-12.

4. Cleuza A, Fontana J, Da Costa S. Sweet diterpenic glycosides balance of a new cultivar of Stevia rebaudiana (Bert.) Bertoni: Isolation and quantitative distribution by chromatographic, spectroscopic, and electrophoretic methods. Process Biochemistry 2005; 40: 3587-3594.

5. Rank A, Midmore D. Stevia an intense natural sweetener: laying the groundwork for a new rural Industries Research and Development Corporation.First edition.RIRDC Edition. Australia. 2006, 12-15.

6. Ramesh K, Singh V, Megeji N. Cultivation of Stevia (Stevia rebaudiana (Bert.) Bertoni): A comprehensive review. Advances in Agronomy 2006; 89 (1): 137 - 170.

7. Brandle J, Rosa N. Heritability for yield, leaf: stem ratio and stevioside content estimated from a landrace cultivar of Stevia rebaudiana. Canadian Journal of Plant Science 1992; 72, 1263-1266.

8. Jiménez D, Montaña J, Martinez M. Characterization of free Nitrogen fixing bacteria of the genus Azotobacter in organic vegetable-grown Colombian Soils. Brazilian Journal of Microbiology 2011; 42: 1-13.

9. Dobbeleare O. The plant growth-promoting effect and plant responses in: Associative and Endophytic Nitrogen-fixing Bacteria and Cyanobacterial Associations. First edition. Springer. Germany. 2007, 145-170.

10. Vivek K, Rishi K, Neeru N. Establishment of phosphatesolubilizing strains of Azotobacter chroococcum in the rizosphere and their effect on wheat cultivars under greenhouse conditions. Microbial Research 2000; 156 (1):87-93.

11. Kumar V, Singh K. Enriching vermi-compost by nitrogen fixing and phosphate solubilizing bacteria. Bioresource Technology 2000; 76: 173-175.

12. Piao Z, Cui Z, Yin B, Hu J, Zhou C, Xie C, Su B, Yin S. Changes in acetylene reduction activities and effects of inoculated rhizosphere nitrogen-fixing bacteria on rice. Biology Fertility Soils 2005; 41: 371-378.

13. Shintani M, Leblanc H, Tabora P. Bokashi, abono orgánico fermentado; Tecnología Tradicional Adaptada para una Agricultura Sostenible y un Manejo de Desechos Modernos. Primera edición. Escuela de Agricultura de la Región Tropical Humedad. San Jose, Costa Rica. 2000, 25.

14. Aslam D, Horwath W, Vandergheynst J. Comparison of several maturity indicators for estimating phytotoxicity in compost-amended soil. Waste Management 2007; 28: 2070-2076.

15. Boulter B, Trevors J, Greg J, Boland C. A polyphasic approach for assessing maturity and stability in compost intended for suppression of plant pathogens. Applied Soil Ecology 2005; 34: 6581-6589. 
16. Loffredo E, Berloco M, Senesi M. The role of humic fractions from soil and compost in controlling the growth in vitro of phytopathogenic and antagonistic soil-borne fungi.Ecotoxicology and Environmental Safety 2008; 69: 350-357.

17. Kovylyaeva G, Bakaleinik A, Yu I, Strobykina I, Gubskaya V, Sharipova R, Alfonsov V, Kataev V, Tolstikov G. Glycosides from Stevia rebaudiana. Chemistry of Natural Compounds 2007; 43 (1): 81-84

18. Margesin R, Schinner L. Manual of Soil Analysis, monitoring and assessing soil bioremediation.Firstedition. SpringerVerlag. Heidejberg, Germany. 2005, 47-93 p.

19. Novo R, Quintana E, Valdes R. Prácticas de Microbiología. Primera edición. Instituto Superior de Ciencias Agropecuarias de la Habana. Facultad de Agronomía. Cuba. 1983, 47-50 p.

20. Borda D, Pardo J, Montaña J, Martínez M. Producción de un biofertilizante a partir de un aislamiento de Azotobacter nigricans obtenido en un cultivo de Stevia rebaudiana Bert. Universitas Scientiarum 2009; 14 (1): 1-8.

21. Maya D. Stevia rebaudiana-Bertoni. Primera edición. Corpoica. Bogotá, D.C. Colombia. 2000, 16 p.

22. Walkley A, Black I. An Examination of Degtjareff Method for Determining Soil Organic Matter and a Proposed Modification of the Chromic Acid Titration Method.Soil Science 1934; 37 (2): 29-37.

23. Tedesco MJ, Gianello C, Bissani C, Volkweiss S. Analisé de solo plantas e outros materiaies. 2. ed. Universidade
Federal do Rio Grande do Sul, Departamento de solos. Porto Alegre, Brazil. 1995, 219 p.

24. Zuberer D, Hartel P, Wollum A. Principles and Applications of Soil Microbiology. Pearson, Prentice Hall. New Jersey. 2005, 8-11 p.

25. Rao I, Rippstein G, Escobar G. Agroecología y biodiversidad de las sabanas en los Llanos Orientales de Colombia. Ed. Ripptein, CIAT. Palmira, Colombia. 2001, 98 p.

26. Pregitzer, King. Effects of soil temperature on nutrient uptake. Ecological Studies 2005; 181, 277-311.

27. Yang M, Shi L, Xu F, Lu J, Wang Y. Effects of B, Mo, $\mathrm{Zn}$ and their interactions on Seed Yield of Rapeseed (Brassica napus L.). Pedosphere 2009; 19 (1): 53 - 59.

28. Ramadass K, Palaniyandi S. Effect of enriched municipal solid waste compost application on soil available macronutrients in the rice field. Archives of Agronomy and Soil Science 2007; 53 (5): 497- 506.

29. Lawrence D, Foster D. Changes in forest biomass, litter dynamics and soils following shifting cultivation in southern México. Interciencia 2002; 27 (8): 400-408.

30. Das K, Dang R, Shivananda T. Influence of biofertilizers on the availability of nutrients $(\mathrm{N}, \mathrm{P} \& \mathrm{~K})$ in soil, in relation to growth and yield of Stevia rebaudiana grown in South India. International Journal of Applied Research in Natural Products 2008; 20-24. 